\title{
Smart antenna technology for structural health monitoring applications
}

\author{
Tayfun Özdemir ${ }^{\mathrm{a}}$, Yuriy Goykhman ${ }^{\mathrm{b}}$, Larry Oberdier*a ${ }^{\mathrm{a}}$, Jerome Lynch ${ }^{\mathrm{bc}}$ \\ ${ }^{\mathrm{a}}$ Monarch Antenna, Inc., Ann Arbor, Michigan, USA; \\ ${ }^{\mathrm{b}}$ Dept. of Electrical Engr. and Computer Sci., University of Michigan, Ann Arbor, Michigan, USA. \\ ${ }^{c}$ Dept. of Civil and Environmental Engr., University of Michigan, Ann Arbor, Michigan, USA.
}

\begin{abstract}
A smart antenna has been developed for structural health monitoring. The antenna is based on Monarch's GEN 2 selfstructuring antenna (SSA) technology and provides polarization and beam-diversity for improving signal-to-noise ratio (SNR). The antenna works with University of Michigan's Narada platform, where a microcontroller monitors the RSSI and selects the best beam to maintain reliable RF link. Antenna has two wide beams for each polarization and the beams are selected by applying appropriate DC voltages to the RF switches on the antenna aperture. Paper presents the GEN C antenna, which is a smaller version of the GEN 2B with comparable performance features.
\end{abstract}

Keywords: Smart Antenna, Structural Health Monitoring, ZigBee, Machine-to-Machine Communication, Wireless Sensor, Mesh Network

\section{INTRODUCTION}

Both polarization diversity and multiple beams are effective ways of increasing Signal-to-Noise-Ratio (SNR) in challenging RF environments [1-5]. Polarization diversity is a cost-effective way to increase SNR using a single aperture $[3,6]$ while employing multiple beams has been shown to consistently increase the channel capacity in low as well as high SNR cases in non-line-of-sight scenarios. Monarch Antenna's patented Self-Structuring Antenna (SSA) technology can be used to provide both polarization diversity and multiple beams, thus enabling more through put in communication systems. Monarch's GEN 2B antenna has been developed to prove this thesis and the details (along with supporting field data) have already been presented in other symposia [7]. This paper presents the development of the GEN $2 \mathrm{C}$ antenna, which is a smaller version of the GEN 2B but is expected to provide similar or better SNR despite its smaller aperture thanks to better impedance matches between the feed lines and the apertures. As in the case of GEN2B, GEN 2C also utilizes the self-structuring feed feature of the Self-Structuring Antenna (SSA) configuration and has four distinct broad beams [8]. Any one of the beams can be selected in an adaptive fashion based on the Received Signal Strength Indicator (RSSI) with the aid of a control algorithm and DC control signals, which drive the RF switches on the antenna aperture. The control algorithm can be a simple set of instructions installed on a micro-controller on the wireless device and 100200 micro-Amperes (at 3Volts) suffice to operate the RF switches.

\subsection{Self Structuring Antenna (SSA)}

Figure 1 shows a regular passive antenna on the left made up of a solid copper (shown in red) connected to a wireless device. SSA aperture on the left is broken into pieces and connected via RF switches, whose on/off states are set by a control algorithm via DC signals. Control algorithm monitors the signal quality (often in terms of RSSI) coming from the device and alters the antenna aperture accordingly to maximize signal quality. The graphics used to represent the SSA aperture (on the right) in Figure 1 should not be taken as a particular antenna design and is used only to draw a contrast with the conventional fixed aperture (shown on the left). SSA technology is well documented in the literature with various applications [8].

*larry@monarchantenna.com; phone 1734 213-4944; fax 1734 661-0159; monarchantenna.com 


\section{Conventional Antenna \\ (Fixed Aperture)}

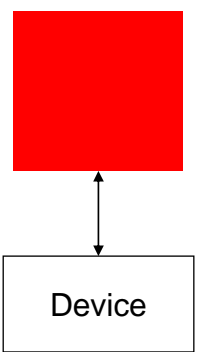

SSA

(Adaptive Aperture)

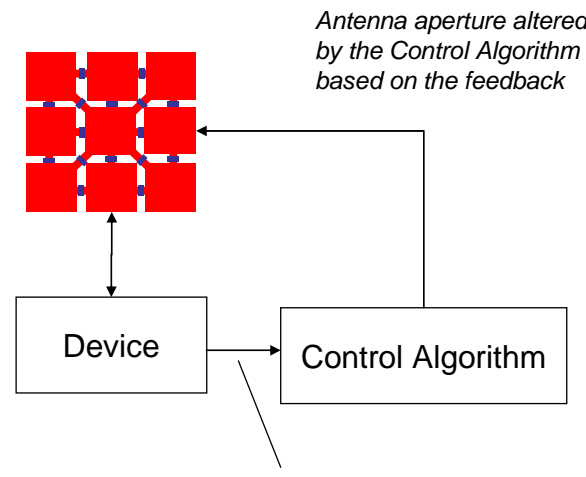

Feedback Signal

Figure 1. A passive antenna on the left and a graphical description of Self-Structuring Antenna (SSA) on the right.

\subsection{GEN 2B}

Figure 1 shows the antenna board with a female SMA connector for RF and a 5-wire connector for DC control (Ground, Supply, and three independent control voltages). The aperture, feed network and the control lines are etched on the top side of a 2-sided PCB board made of 0.093" $(2.36 \mathrm{~mm})$ thick Isola FR408 laminate with 1oz copper deposit on both sides in a microstrip arrangement. The horizontal dimensions are $154 \mathrm{~mm} \times 154 \mathrm{~mm}$. Backside of the antenna is solid copper and is responsible for the hemi-spherical coverage. Antenna has a broad high gain pattern toward the side of the board, on which the aperture is etched and a low gain pattern toward the back of the board (which is solid copper). Measured Front-To-Back Ratio (FBR) is $13 \mathrm{~dB}$, which contributes about $3 \mathrm{~dB}$ increase to the front-side gain. The Antenna is capable of radiation and reception at either vertical or horizontal polarization with two beams per polarization. The maximum gain for beams varies between 1 and $3 \mathrm{dBi}$. The beam switching operation is accomplished via a control software running on a laptop and pushing out control DC signals through a USB adapter. When integrating the GEN 2B into a wireless device, this control algorithm would reside in a microcontroller.

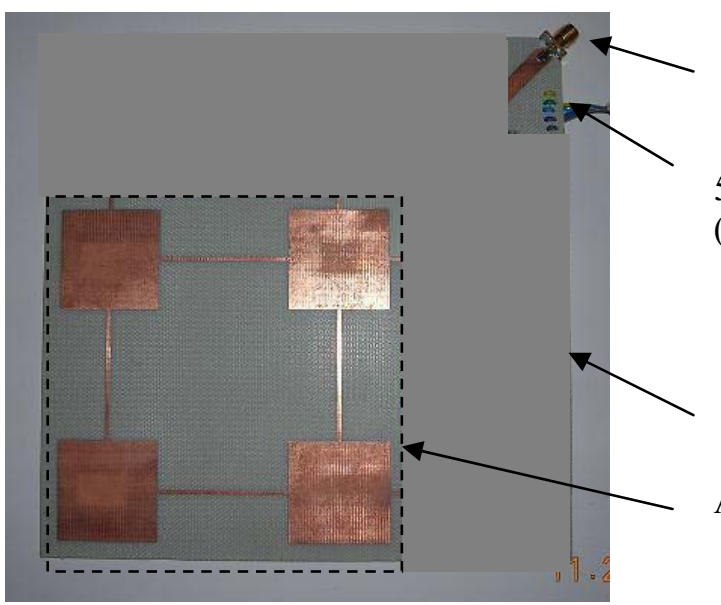

(a)

\section{SMA Connector (RF Signal) \\ 5-wire Connector (DC Control Signals)}

Feed Network, FET switches (hidden)

\section{Aperture}

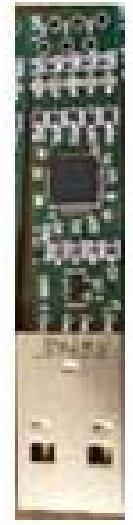

(b)

Figure 2. (a) GEN 2B antenna printed on 0.093" (2.39mm) FR4 (Isola FR408) with nominal parameters of $\mathrm{r}=3.77$ and $\tan .=0.012$. Copper cladding is $0.5 \mathrm{oz}$ on each side. The feed network is not shown due to proprietary nature of the design, (b) USB adaptor used to generate DC control signals (3V) from a control algorithm running on a laptop. 
GEN 2B was tested in a machine-to-machine communication scenario using commercial-off-the-shelf ZigBee units [9]. The data collection and the control of the antenna were accomplished via two software running simultaneously on a laptop: One software pulled the RSSI data from both the Coordinator and the End Unit while the second software generated DC control signals to switch among the four independent beams. Figure 3 shows the floor plan of the second floor of Monarch's old facility in Belleville, Michigan, where the Controller was placed in the Conference room while the End Unit was moved from one location to another (Storage 1, 2 and Office 1,2,3) to record the RSSI. First, both units had monopoles connected to them while the End Unit was placed such that the monopole is vertical (aligned with the Controller) and also horizontal (not aligned with the Controller). For both the vertical and horizontal cases, the RSSI was recorded. In the next step, the Controller was connected to the GEN 2 antenna along with all the interface hardware needed to control the antenna while the monopole remained on the End Unit. For each of the End Unit locations and for each orientation of its monopole (vertical and horizontal), RSSI was observed for each of the four beams of GEN 2 and the highest RSSI value was recorded (one of the four beams always gave the highest RSSI). The values indicated on the floor plan in Figure 3 are the improvements in RSSI when GEN 2 is connected to the Controller (as opposed to the monopole) for the vertical $(\mathrm{V})$ and the horizontal $(\mathrm{H})$ orientations of the End Unit's monopole. The improvement is significant with an average value of $9.4 \mathrm{~dB}$. The difference is more pronounced when the End Unit is horizontally placed since this represents a polarization mismatch for the monopole-to-monopole case while the GEN 2 is able to correct its polarization in the GEN 2-to-monopole case.

\begin{tabular}{|c|c|c|c|c|}
\hline $\begin{array}{l}\text { Conference } \\
\text { Room } \\
\text { (Controller) }\end{array}$ & \multirow{3}{*}{ 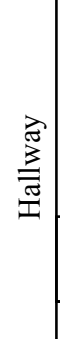 } & & \multirow[t]{2}{*}{ Storage } & \\
\hline \multirow{2}{*}{$\begin{array}{c}\text { Storage } 1 \\
\begin{array}{c}\mathbf{V}=\mathbf{6 d B} \\
\mathbf{H}=\mathbf{1 3 d B}\end{array}\end{array}$} & & Stair & & \\
\hline & & \multicolumn{3}{|c|}{ Hallway } \\
\hline Office 1 & & Office 2 & Storage 2 & Office 3 \\
\hline $\begin{array}{l}V=7 d B \\
H=7 d B\end{array}$ & & $\begin{array}{l}V=13 \mathbf{d B} \\
H=5 \mathbf{d B}\end{array}$ & $\begin{aligned} V & =3 \mathrm{DB} \\
\mathrm{H} & =\mathbf{1 4 d B}\end{aligned}$ & $\begin{array}{l}V=12 \mathrm{~dB} \\
H=14 \mathrm{~dB}\end{array}$ \\
\hline
\end{tabular}

Fig. 3. Floor plan of the second floor of Monarch's facility where the indoor tests were conducted, showing the improvement in RSSI by the GEN 2 antenna over the monopole in units of dBm. Average improvement is 9.4dB.

\section{GEN 2C}

\subsection{Aperture}

Aperture of GEN 2C antenna is the same as that of GEN 2B except for the microstrip lines connecting the patches to each other as shown in Figure 4. From GEN 2B to GEN 2C, the microstrip lines went from straight to meanderline reducing the spacing between the patches and hence shaving off 0.75 " from the horizontal dimension. As this is done, the impedance of the aperture changed, so the spacing between the patches and the meander line sections had to be adjusted using simulations, resulting in good match with the feed. Good agreement between simulation and measurement was achieved as evident in Table 1, which shows data for resonance frequency and input impedance of $2 \times 2$ patches (the aperture only). The feed network is currently being designed and will not be discussed here due to its proprietary nature. Figure 5 shows the co-pol measured gain patterns of the $2 \times 2$ aperture shown in Figure 4(b). Maximum gains are 6.4dBi and $6.8 \mathrm{dBi}$ in the E-Plane and H-Plane, respectively. Figure 6 shows the $\mathrm{X}$-pol gain patterns and the maximum gains are $-9 \mathrm{dBi}$ and $-8.6 \mathrm{dBi}$ in the E-Plane and H-Plane, respectively. X-polarization isolation of $15.4 \mathrm{~dB}$ (in both E- and H-planes) is an excellent result for a polarization diversity antenna. 
Measurements are taken at frequencies where the maximum gain is recorded along a particular cut. The frequencies are close to each other (for co-pol vs. X-pol or E-Plane vs. H-Plane) but different nevertheless. It is important to observe that the maximum gain for co-pol gain is recorded at $2,470 \mathrm{MHz}$ (see Figure 5), which is almost identical to the measured frequency at which the antenna resonates (see Table 1). The significance of this is that the feed of the antenna (see Figure $4 \mathrm{~b}$ ) is matched correctly and that the patch radiators (which make up the GEN 2C's aperture) are driven at their resonance frequencies, i.e., are operating at their peak efficiency.

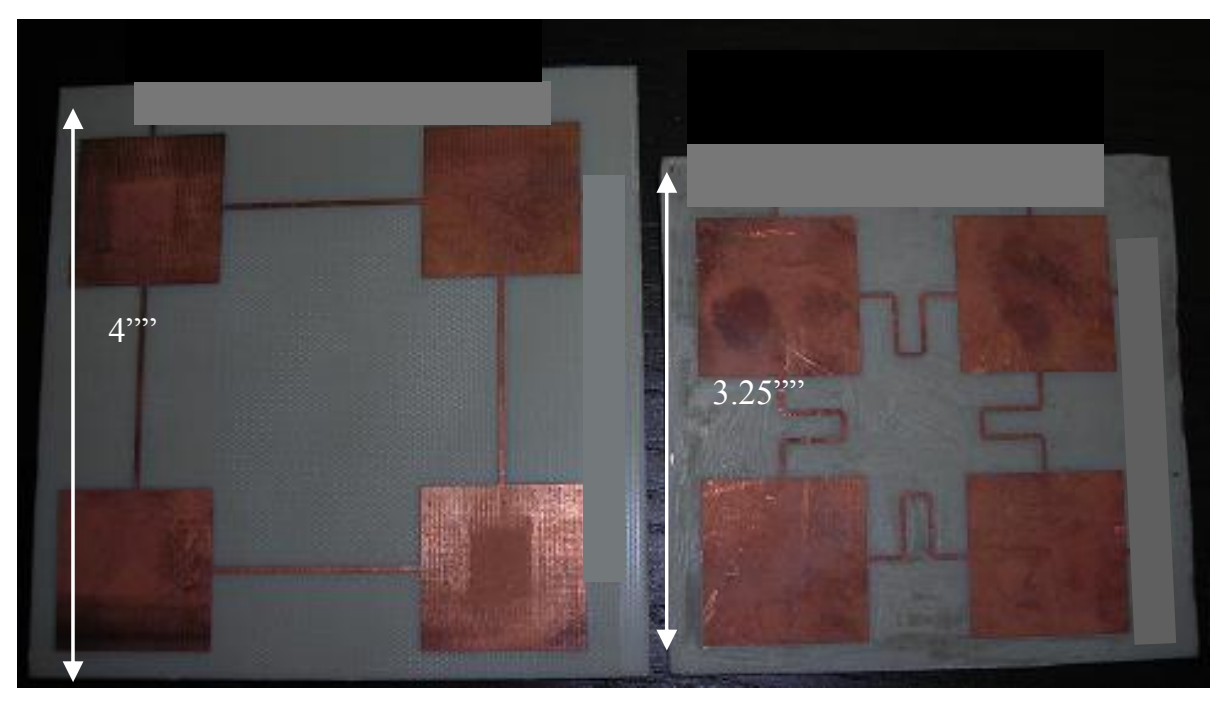

(a)

(b)

Figure 4. Aperture of (a) GEN 2B and (b) GEN 2C. Feed network of is hidden due to proprietary nature of the design.

Table 1. Simulated and measured resonance frequencies and input impedances of the $2 \times 2$ antenna aperture (excluding the feed network).

\begin{tabular}{|l|l|l|}
\hline & Simulation & Measurement \\
\hline Resonant Frequency & $2.547 \mathrm{GHz}$ & $2.474 \mathrm{GHz}$ \\
\hline Impedance & $43 \mathrm{Ohms}$ & $34.5 \mathrm{Ohms}$ \\
\hline
\end{tabular}

\subsection{Control Signals and Integration with the Narada Platform}

Narada Platform is a ZigBee-based communication node providing data collection from sensors and processing in a wireless mesh network environment. The GEN 2C antenna has been connected to the Narada board as shown in 7(a) and is ready for indoor tests. A ribbon cable carries DC control signals (5 Volts) from Narada to GEN 2C and an RF cable provides RF signal to Narada from the GEN $2 \mathrm{C}$ board. The ribbon connectors chosen for this are produced by Tyco and consist of a board mounted low-profile connector and a polarized ribbon mounted mating connector with interference fit. At the Narada end of the cable, it was necessary to build an adapter board since no standard ribbon connector is available for the Samtec connector on the Narada. Initially, protoboard with 50 mil hole spacing was used to construct the adapter for the first system tests as shown in 67b), which will eventually be replaced by a small printed circuit board adapter. The control signals carried by the ribbon cable consist of a 5.0 VDC power, one ground and two 0 vs. 5 VDC logic lines to drive the switches on the feed network for polarization selection and beam steering. 
A lean and efficient antenna control algorithm has already been developed and tested successfully using GEN 2B antenna and commercial ZigBee wireless units (see Section 1.2 above). The algorithm will be next converted from C++ to $\mathrm{C}$ and ported to the micro-controller on the Narada board. The algorithm uses a simple search algorithm to look for a better polarization-beam combination when the Received Signal Strength Indicator (RSSI) provided by the Narada radio drops below a pre-set threshold (usually a value between -45 to $-60 \mathrm{dBm}$ ).
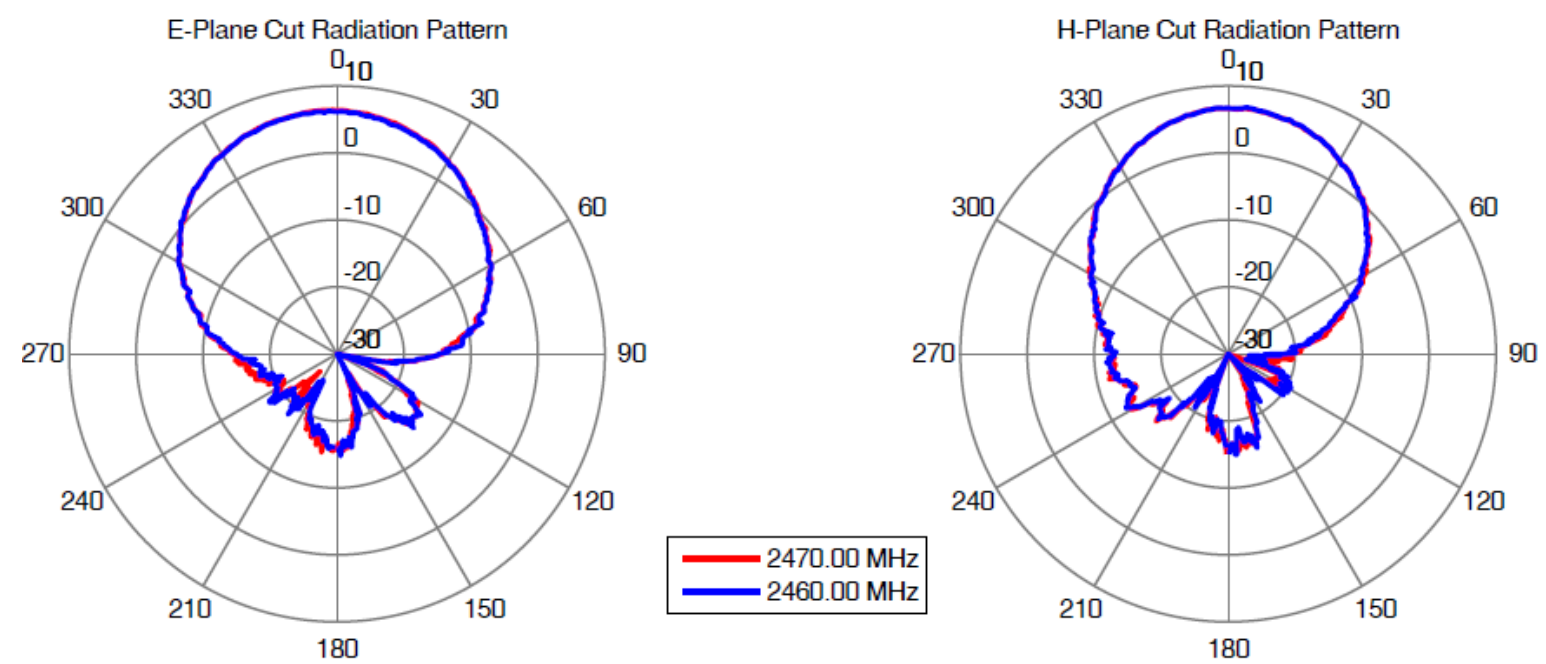

Figure 5. Co-pol measured gain patterns of the 2x2 aperture shown in Figure 4(b). Maximum gains are 6.4dBi and 6.8dBi in the E-Plane and H-Plane, respectively.
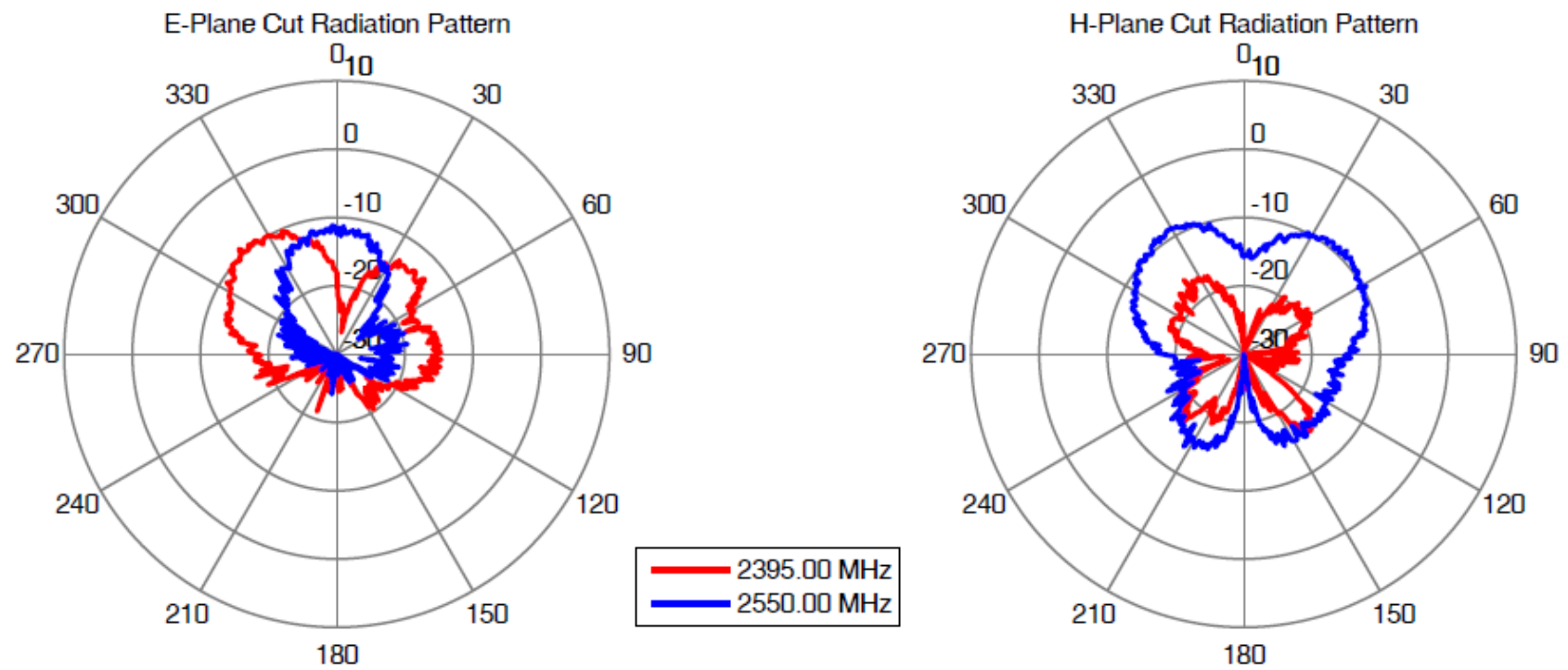

Figure 6. X-pol measured gain patterns of the $2 \times 2$ aperture shown in Figure 4(b). Maximum gains are $-9 \mathrm{dBi}$ and $-8.6 \mathrm{dBi}$ in the E-Plane and H-Plane, respectively. 


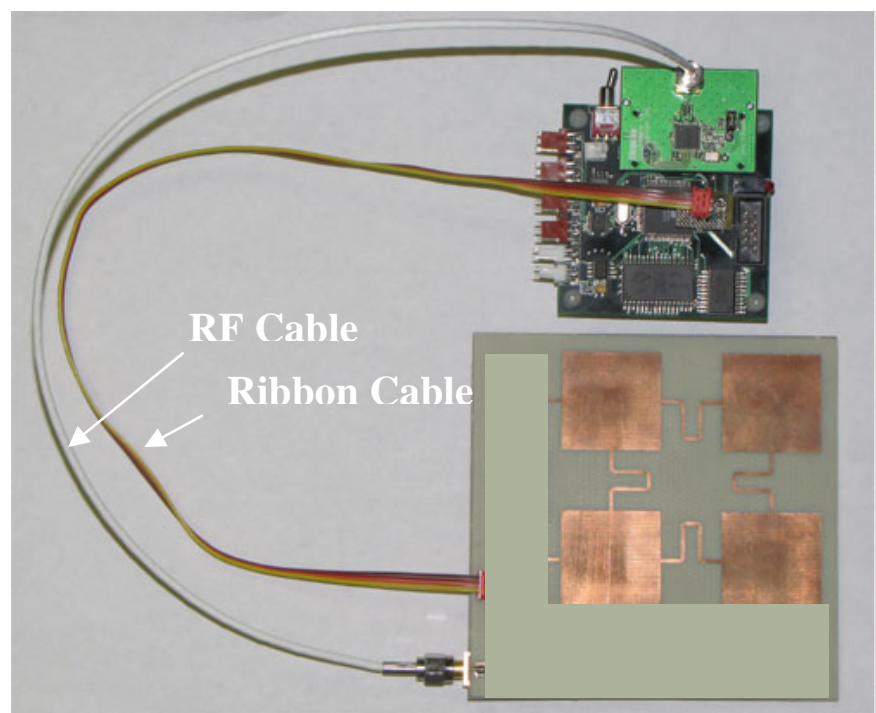

(a)

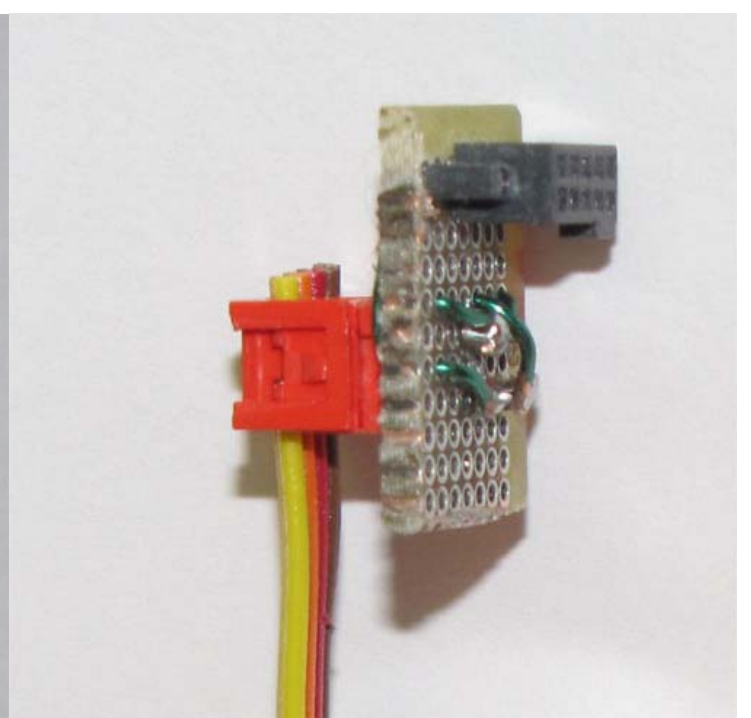

(b)

Figure 7. (a) Narada and GEN $2 \mathrm{C}$ antenna are connected via a ribbon cable (for control signals) and RF cable, (b) a small adapter board built using 50 mil protoboard is used for mating between the ribbon cable and the connecter on the Narada board.

\section{REMAINING WORK}

\subsection{Feed Network}

The feed network of the GEN 2B and GEN 2C use the same principal for beam steering. However, GEN 2C will have a more efficient feed network, achieving the same amount of beam steering (about 20 degrees) while occupying 1" less space in both horizontal dimensions. In subsequent iterations, there are plans for moving the feed network to a second layer to completely eliminate it from the top layer, hence resulting in an even smaller antenna (smaller than 3" in both horizontal dimensions). Feed network of GEN $2 \mathrm{C}$ is currently being finalized, and it will be followed by beam steering measurements. Unfortunately, the paper will not be able to discuss the details of the feed network design due to its proprietary nature.

\subsection{Indoor Tests}

After the GEN 2C feed network is finalized, indoor tests that are similar to the ones performed for GEN 2B (see Section 1.2) will be conducted by comparing the performance of the GEN $2 \mathrm{C}$ to a monopole using Narada nodes.

\subsection{Outdoor Tests}

Tests will be repeated on an actual bridge structure donated by Michigan Department of Transportation (MDOT), specifically the Grove Street/I94 Bridge in Southeast Michigan.

\section{CONCLUSIONS}

The paper describes the development of the GEN 2C antenna, which is a smart antenna designed to be used in structural health monitoring applications. GEN $2 \mathrm{C}$ is a smaller version of the GEN $2 \mathrm{~B}$ but with similar capabilities and is designed to be integrated into Narada wireless sensor nodes. Narada nodes provide sensor data collection and routing in a mesh 
environment using ZigBee and GEN 2C will replace the existing monopole antenna in order to improve signal-to-noise ratio (SNR) through polarization and beam diversity.

\section{ACKNOWLEDGEMENTS}

The authors would like to gratefully acknowledge the generous support offered by the U.S. Department of Commerce, National Institute of Standards and Technology (NIST) Technology Innovation Program (TIP) under Cooperative Agreement Number 70NANB9H9008. Additional support was provided by Monarch Antenna, Inc., Delphi Corporation and Virtual EM Inc.

\section{REFERENCES}

[1] Cover, T. M., and Thomas, J. A., [Elements of Information Theory], John Wiley \& Sons, New York (2006).

[2] Cui, S., Goldsmith, A. J. and Bahai, A. "Energy-efficiency of MIMO and Cooperative MIMO in Sensor Networks," IEEE J. Select. Areas of Commun. 22(6), 1089-1098 (2004).

[3] Zhao, Z., Stapleton, S. and Cavers, J. K., "Analysis of polarization diversity scheme with channel codes," 1999 IEEE Vehicular Technology Conference, Fall-1999, 3, 1377-1381 (1999).

[4] Neasmith, E. A. and Beaulieu, N.C., "New results on selection diversity," IEEE Trans. Comm. 46(5), 695-704 (1998).

[5] Gurrieri, L.E. and Noghanian, S., "Multibeam Selection for Indoor MIMO Systems: Two Cases of Study," IEEE Trans. Antenna Propagat., 55(4), 1173-1179 (2007).

[6] Ozdemir, T., "Polarization Diversity Cognitive Antenna for WiFi and ZigBee Applications," WAMICON 2009, Clear Water, FL (2009).

[7] Ozdemir, T., "A Polarization Diversity Multi-Beam Antenna for ZigBee Applications," WAMICON 2009, Clear Water, FL (2009).

[8] Coleman, C. M., Rothwell, E. J., Ross, J. E., and Nagy, L. L., "Self-Structuring Antennas," IEEE Antennas Propagat. Mag., 44(3), 11-22 (2002).

[9] http://www.meshnetics.com/dev-tools/meshbean/. 